\title{
FIXED POINTS OF CERTAIN INVOLUTIONS IN BANACH SPACES
}

\author{
M. S. KHAN and M. IMDAD
}

(Received January 5, 1983)

Communicated by J. H. Rubinstein

\begin{abstract}
Some results on fixed points of certain involutions in Banach spaces have been obtained, and whence a few coincidence theorems are also derived. These are indeed generalization of previously known results due to Browder, Goebel-Zlotkiewicz and Iséki. Illustrative examples are also given.

1980 Mathematics subject classification (Amer. Math. Soc.): primary 54 H 25; secondary 46 B 99. Keywords and phrases: involution, Banach space, convex set, fixed point, coincidence point, modulus of convexity.
\end{abstract}

\section{Introduction}

Let $X$ be a Banach space and $C$ a closed subset of $X$. Then the well-known Banach Contraction Principle states that a contraction mapping of $C$ into itself has a unique fixed point. The same conclusion holds good if we assume that only some positive power of a mapping is a contraction (for example, Bryant [2]). But this result is no longer true for nonexpansive mappings. Many mathematicians have studied the existence of fixed points of nonexpansive maps defined on a closed, bounded and convex subset of a uniformly convex Banach space, and in a space with a normal structure. For the results of this kind one is referred to Browder [1], Goebel [4] and Kirk [8]. It is natural to ask the question whether these results can be extended to mappings with a nonexpansive iteration. The answer, in general, is negative (for example, Klee [9]). However Goebel and Zlotkiewicz [5] have answered this problem in affirmative with some restriction,

(c) 1984 Australian Mathematical Society 0263-6115/84 \$A2.00+0.00 
and thus generalizing a result of Browder [1]. Recently, Iséki [7] obtained a generalization of Goebel-Zlotkiewicz's result by employing a contractive condition of Reich [10].

The purpose of this paper is to present yet another extension of a result due to Goebel-Zlotkiewicz [5] which in turn generalizes the main result of Iséki [7]. These results are also used to obtain some coincidence theorems. Some examples are provided to justify our results. Of course, our results are motivated by Ciric [3].

\section{Results}

Throughout this section $X$ stands for a Banach space and $I$ denotes the identity mapping on $X$.

THEOREM 2.1. Let $C$ be a closed and convex subset of $X$ and let $E: C \rightarrow C$ satisfy the conditions

(i) $E^{2}=I$,

(ii)

$$
\begin{aligned}
\|E x-E y\| \leqslant(\alpha / 2) \max \left\{\|x-y\|, \frac{1}{2}\|x-E x\|,\right. \\
\\
\left.\frac{1}{2}\|y-E y\|, \frac{1}{2}\|x-E y\|, \frac{1}{2}\|y-E x\|\right\}
\end{aligned}
$$

for every $x, y \in C$, where $0 \leqslant \alpha<4$. Then $E$ has at least one fixed point.

Proof. Let $x$ be an arbitrary point of $C$ and $H=\frac{1}{2}(I+E)$. Put $y=H x$, $z=E y$ and $u=2 y-z$. Then we have

$$
\begin{aligned}
\|z-x\| & =\left\|E y-E^{2} x\right\| \\
& \leqslant(\alpha / 2) \max \left\{\|y-E x\|, \frac{1}{2}\|y-E y\|, \frac{1}{2}\|E x-x\|,\right. \\
& =(\alpha / 4) \max \{\|x-E x\|,\|y-E y\|\}
\end{aligned}
$$

and

$$
\|u-x\|=\|E x-E y\| \leqslant(\alpha / 4) \max \{\|x-E x\|,\|y-E y\|\} .
$$

Hence

$$
\|z-u\| \leqslant(\alpha / 2) \max \{\|x-E x\|,\|y-E y\|\} .
$$

On the other hand, we have $\|z-u\|=2\|y-E y\|$. Therefore

$$
\|y-E y\| \leqslant(\alpha / 4) \max \{\|x-E x\|,\|y-E y\|\} .
$$


If $\|y-E y\| \leqslant(\alpha / 4)\|y-E y\|$, then $y$ is a fixed point of $E$. Note that $y \in C$ since $C$ is convex.

If $\|y-E y\| \leqslant(\alpha / 4)\|x-E x\|$, then

$$
\left\|H^{2} x-H x\right\|=\frac{1}{2}\|y-E y\| \leqslant(\alpha / 8)\|x-E x\|=(\alpha / 4)\|H x-x\| .
$$

Since $0 \leqslant \alpha / 4<1$, the sequence $\left\{x_{n}\right\}$ defined by $x_{n}=H^{n} x$ converges. Put $x^{*}=\lim _{n \rightarrow \infty} x_{n}$. Now

$$
\begin{aligned}
&\left\|x^{*}-H x^{*}\right\| \leqslant\left\|x^{*}-x_{n+1}\right\|+\left\|H x_{n}-H x^{*}\right\| \\
&=\left\|x^{*}-x_{n+1}\right\|+\left\|\frac{1}{2}\left(x_{n}+E x_{n}\right)-\frac{1}{2}\left(x^{*}+E x^{*}\right)\right\| \\
& \leqslant\left\|x^{*}-x_{n+1}\right\|+\frac{1}{2}\left\|x_{n}-x^{*}\right\|+\frac{1}{2}\left\|E x_{n}-E x^{*}\right\| \\
& \leqslant\left\|x^{*}-x_{n+1}\right\|+\frac{1}{2}\left\|x_{n}-x^{*}\right\| \\
&+(\alpha / 4) \max \left\{\left\|x_{n}-x^{*}\right\|, \frac{1}{2}\left\|x_{n}-E x_{n}\right\|,\right. \\
&\left.\frac{1}{2}\left\|x^{*}-E x^{*}\right\|, \frac{1}{2}\left\|x_{n}-E x^{*}\right\|, \frac{1}{2}\left\|x^{*}-E x_{n}\right\|\right\} \\
& \leqslant\left\|x^{*}-x_{n+1}\right\|+\frac{1}{2}\left\|x_{n}-x^{*}\right\| \\
&+(\alpha / 4) \max \left\{\left\|x_{n}-x^{*}\right\|, \frac{1}{2}\left\|x_{n}-E x_{n}\right\|,\right. \\
& \quad \frac{1}{2}\left\|x^{*}-E x^{*}\right\|, \frac{1}{2}\left(\left\|x_{n}-x^{*}\right\|+\left\|x^{*}-E x^{*}\right\|\right), \\
&=\left\|x^{*}-x_{n+1}\right\|+\frac{1}{2}\left\|x_{n}-x^{*}\right\| \\
&+(\alpha / 4) \max \left\{\left\|x_{n}-x^{*}\right\|,\left\|x_{n+1}-x_{n}\right\|,\right. \\
&\left\|H x^{*}-x^{*}\right\|,\left(\frac{1}{2}\left\|x_{n}-x^{*}\right\|+\left\|H x_{n}^{*}-x^{*}\right\|\right), \\
&\left.\quad\left(\frac{1}{2}\left\|x^{*}-x_{n}\right\|+\left\|x_{n+1}-x_{n}\right\|\right)\right\} .
\end{aligned}
$$

Letting $n \rightarrow \infty$, we get

$$
\left\|x^{*}-H x^{*}\right\| \leqslant(\alpha / 4)\left\|x^{*}-H x^{*}\right\| .
$$

As $\alpha<4$, we have $x^{*}=H x^{*}$. Hence $x^{*}=E x^{*}$. This completes the proof.

As an immediate consequence of Theorem 2.1, we have the following result which presents a slightly improved version of the only theorem of Iséki [7].

COROLlaRY 2.2. Let $E$ be a mapping of $X$ into itself. If $E$ satisfies the conditions

(i) $E^{2}=I$,

(ii) $\|E x-E y\| \leqslant \alpha\|x-y\|+\beta(\|x-E x\|+\|y-E y\|)+\gamma(\|x-E y\|$ $+\|y-E x\|)$

for every $x, y \in X$, where $0 \leqslant \alpha, \beta, \gamma$ and $0 \leqslant \alpha+4 \beta+4 \gamma<2$, then $E$ has at least one fixed point. 
Proof. For all $x, y \in X$, we have

$$
\begin{aligned}
\|E x-E y\| \leqslant & \alpha\|x-y\|+2 \beta\left(\frac{1}{2}\|x-E x\|+\frac{1}{2}\|y-E y\|\right) \\
& +2 \gamma\left(\frac{1}{2}\|x-E y\|+\frac{1}{2}\|y-E x\|\right) \\
\leqslant & (\alpha+4 \beta+4 \gamma) \max \left\{\|x-y\|, \frac{1}{2}\|x-E x\|, \frac{1}{2}\|y-E y\|,\right. \\
& \left.\frac{1}{2}\|x-E y\|, \frac{1}{2}\|y-E x\|\right\} .
\end{aligned}
$$

The result now follows from Theorem 2.1.

REMARKs. (a) It would be of interest to extend Theorem 2.1 to the case when $C$ is a starshaped subset of a Banach space $X$.

(b) If $\gamma=0$ in Corollary 2.2, we get the result of Iséki [7].

(c) For $\beta=\gamma=0$, the Corollary 2.2 reduces to Theorem 1 of Goebel and Zlotkiewicz [5].

(d) Observe that condition (ii) of Corollary 2.2 was first introduced by Hardy and Rogers [6].

Now we apply Theorem 2.1 to prove a coincidence theorem.

Theorfm 2.3. Let $C$ be a closed and convex subset of $X$. Let $E: C \rightarrow C$ and $F$ : $C \rightarrow C$ satisfy the following conditions:

(i) $E^{2}=I, F^{2}=I$,

(ii)

$$
\begin{aligned}
&\|E x-E y\| \leqslant(\alpha / 2) \max \left\{\|F x-F y\|, \frac{1}{2}\|F x-E x\|, \frac{1}{2}\|F y-E y\|,\right. \\
&\left.\frac{1}{2}\|F x-E y\|, \frac{1}{2}\|F y-E x\|\right\}
\end{aligned}
$$

for every $x, y \in C$, where $0 \leqslant \alpha<4$.

Then there exists at least one point $x_{0} \in C$ such that $E x_{0}=F x_{0}$.

Proof. From Theorem 2.1 there exists at least one point $x_{0} \in C$ such that $E F x_{0}=x_{0}$. Then $E x_{0}=F x_{0}$.

ReMARK. If $0 \leqslant \alpha<2$ then $x_{0}$ is indeed a common fixed point of $E$ and $F$.

The next two results are slight generalizations of Theorem 2 and Theorem 3 of Goebel and Zlotkiewicz [5]. 
THEOREM 2.4. Let $X$ be a uniformly convex space and $C$ a closed and convex subset of $X$. Then each mapping $E: C \rightarrow C$ satisfying (i) of Theorem 2.1 and also the inequality

(ii)

$$
\|E x-E y\| \leqslant \alpha \max \left\{\|x-y\|, \frac{1}{2}\|x-E x\|\right\},
$$

for all $x, y \in C$, with $\alpha$ such that $\alpha \delta^{-1}(1-1 / \alpha)<4$ ( $\delta$ denotes the modulus of convexity of $X$ ), has at least one fixed point.

In the sequel $\alpha$ satisfies the condition of Theorem 2.4 .

THEOREM 2.5. Let $X$ be a uniformly convex space and $C$ is closed, bounded and convex subset of $X$. Let $E: C \rightarrow C$ satisfy the conditions

(i) $\left\|E^{2} x-E^{2} y\right\| \leqslant\|x-y\|$,

(ii) $\|E x-E y\| \leqslant \alpha \max \left\{\|x-y\|, \frac{1}{2}\|x-E x\|\right\}$, for all $x, y \in C$. Then there is at least one fixed point of $E$.

The following are the coincidence theorems corresponding to Theorem 2.4 and Theorem 2.5 respectively.

THEOREM 2.6. Let $X$ be a uniformly convex space and $C$ a closed and convex subset of $X$. Consider the pair of mappings $E: c \rightarrow C$ and $F: c \rightarrow C$ satisfying

(i) $E^{2}=I, F^{2}=I$,

(ii) $\|E x-E y\| \leqslant \alpha \max \left\{\|F x-F y\|, \frac{1}{2}\|F x-E x\|\right\}$, for all $x, y \in C$.

Then there exists at least one point $x_{0} \in C$ such that $E x_{0}=F x_{0}$.

Theorem 2.7. Let $X$ be a uniformly convex space and $C$ a closed, bounded and convex subset of $X$. Let $E: C \rightarrow C$ and $F: C \rightarrow C$ satisfy condition (ii) of Theorem 2.6 and further

(i) $F^{2}=I$,

(ii) $E$ and $F$ commute,

(iii) $\left\|E^{2} x-E^{2} y\right\| \leqslant\|x-y\|$.

Then $E$ and $F$ have at least one point of coincidence.

Proof. Since $E^{2}$ is nonexpensive, by Browder's theorem [1], $E^{2}$ has at least one fixed point in $C$. Then the set $C^{*}=\left\{x \in C: E^{2} x=x\right\}$ is closed and convex. As $E$ and $F$ commute, $F$ maps $C^{*}$ into itself. Also $E^{2}=I$ on $C^{*}$. Hence Theorem 2.6 pertains to $C^{*}$. This ends the proof. 
REMARK. The conclusion of Theorem 2.4 and Theorem 2.6 still holds if condition (ii) is replaced by the following:

(*)

$$
\begin{array}{r}
\|E x-E y\| \leqslant \alpha \max \left\{\|x-y\|, \frac{1}{2}\|x-E x\|, \frac{1}{2}\|y-E y\|,\right. \\
\left.\frac{1}{2}\|x-E y\|, \frac{1}{2}\|y-E x\|\right\},
\end{array}
$$

and

$(* *)$

$$
\max \{\|y-E y\|,\|x-E y\|,\|y-E x\|\} \leqslant \inf \|x-E x\|
$$

where $x, y \in C$.

It would be of interest to know whether the condition (**) is actually needed.

\section{Some related examples}

In this section, we furnish examples to discuss the validity of the hypotheses and degree of generality of our results.

EXAmple (3.1). This example shows that our Theorem 2.1 is a genuine extension of the main result of Iséki [7].

Let $C=X=R$, the set of reals with usual norm, and $E: R \rightarrow R$ is defined as follows:

$$
E x= \begin{cases}-20 x & \text { if } x \geqslant 0 \\ -\frac{1}{20} x & \text { if } x<0\end{cases}
$$

Note that $E^{2}=I$, the identity map of $R$. Then for $x<0$ and $y<0$, we have

$$
|E x-E y|=\frac{1}{20}|x-y| \leqslant \frac{82}{42}|x-y|=\frac{1}{2} \cdot \frac{82}{21}|x-y|
$$

whereas for $x \geqslant 0$ and $y \geqslant 0$, we get

$$
|E x-E y|=20|x-y| \leqslant \begin{cases}\frac{20}{21} \cdot 21 x & \text { if } x \geqslant y \\ \frac{20}{21} \cdot 21 y & \text { if } x \leqslant y\end{cases}
$$

Thus in both the cases, we find that

$$
\begin{aligned}
|E x-E y| & \leqslant \frac{20}{21} \max \{21 x, 21 y\}=\frac{20}{21}\{|x-E x|,|y-E y|\} \\
& <\frac{1}{2} \cdot \frac{82}{21} \max \left\{\frac{1}{2}|x-E x|, \frac{1}{2}|y-E y|\right\} .
\end{aligned}
$$

Furthermore, for $x \geqslant 0$ and $y<0$, we have if $-y \leqslant 10 x$ :

$$
\begin{aligned}
|E x-E y| & =20 x+\frac{1}{20}(-y) \leqslant 20 x+\frac{1}{20} 10 x=\frac{41}{2 \cdot 21} 21 x \\
& =\frac{1}{2} \cdot \frac{82}{21} \cdot \frac{1}{2} 21 x=\frac{1}{2} \cdot \frac{82}{21} \cdot \frac{1}{2}|x-E x| .
\end{aligned}
$$


Next, if $-y>10 x$, the following sequence of implications holds:

$$
\begin{aligned}
-y>10 x & \Rightarrow-799 y>7990 x>7580 x \\
& \Rightarrow 21 y-820 y>8400 x-820 x \Rightarrow 820 x-820 y>8400 x-21 y \\
& \Rightarrow \frac{820}{21}(x-y)>400 x-y \Rightarrow \frac{1}{2} \cdot \frac{82}{21}(x-y)>20 x-\frac{1}{20} y \\
& \Rightarrow \frac{1}{2} \cdot \frac{82}{21}|x-y|>20 x+\frac{1}{20}(-y)=E x-E y .
\end{aligned}
$$

By summarising, for any $x, y \in R$, we found that

$$
|E x-E y| \leqslant \frac{1}{2} \cdot \frac{82}{21} \cdot \max \left\{|x-y|, \frac{1}{2}|x-E x|, \frac{1}{2}|y-E y|\right\} .
$$

Thus condition (ii) of Theorem 2.1 is satisfied for the choice $\alpha=\frac{82}{21}$. Here $x=0$ is the only fixed point of $E$.

However, condition (ii) of Corollary 2.2 is not satisfied. Otherwise, for $x=0$ and $y=1$, we should obtain

$$
\begin{aligned}
20 & \leqslant \alpha+\beta(0+21)+\gamma(20+1)=\alpha+21 \beta+21 \gamma \\
& \leqslant \frac{21}{4} \alpha+21 \beta+21 \gamma
\end{aligned}
$$

which is a contradiction because from $\alpha+4 \beta+4 \gamma<2$, it follows that $\frac{21}{4} \alpha+21 \beta$ $+21 \gamma<\frac{21}{2}<20$.

Of course, this example is also not satisfied by mappings considered by Iséki [7].

EXAMPLE (3.2). We are facing some difficulties while constructing two self-maps of $R$ which strictly satisfy condition (ii) of Theorem 2.3. However, we have found two self-maps $E$ and $F$ on $R$ which satisfy the condition

$$
|E x-E y| \leqslant(\alpha / 2)|F x-F y| \text {. }
$$

Condition (ii) of Theorem 2.3 is clearly more general than (A), and hence the following example can be adopted in order to support condition (ii) of Theorem 2.3. Condition (A) also seems to be new in Fixed Point Theory.

Let $X=C=R$, with usual norm. Define $E: R \rightarrow R, F: R \rightarrow R$ as follows:

$$
E x=\left\{\begin{array}{ll}
-3 x & \text { if } x \geqslant 0, \\
-\frac{1}{3} x & \text { if } x<0,
\end{array} \quad F x= \begin{cases}-4 x & \text { if } x \geqslant 0 \\
-\frac{1}{4} x & \text { if } x<0\end{cases}\right.
$$

Observe that $E^{2}=F^{2}=$ identity map on $R$. For $x \geqslant 0, y \geqslant 0$ we have

$$
\begin{aligned}
|E x-E y| & =3|x-y| \leqslant \frac{7}{8} \cdot 8|x-y|=\frac{1}{2} \cdot \frac{28}{8} \cdot 4|x-y| \\
& =\frac{1}{2} \cdot \frac{28}{8}|F x-F y|
\end{aligned}
$$


and when $x<0, y<0$ we obtain

$$
\begin{aligned}
|E x-E y| & =\frac{1}{3}|x-y| \leqslant \frac{7}{16}|x-y|=\frac{1}{2} \cdot \frac{28}{8} \cdot \frac{1}{4}|x-y| \\
& =\frac{1}{2} \cdot \frac{28}{8}|F x-F y| .
\end{aligned}
$$

Next, for $x \geqslant 0$ and $y<0$, we write a sequence of implication in the following way:

$$
\begin{aligned}
y<0 \leqslant x & \Rightarrow y<\frac{192}{5} x \Rightarrow y<\frac{48}{5} \cdot 4 x \Rightarrow \frac{5}{48} y<4 x \\
& \Rightarrow-\frac{7}{16} y-\frac{1}{3} y<7 x-3 x \Rightarrow 3 s x-\frac{1}{3}(-y)<7 x+\frac{7}{16}(-y) \\
& \Rightarrow 3 x+\frac{1}{3}(-y)<\frac{14}{8}\left[4 x+\frac{1}{4}(-y)\right]=\frac{1}{2} \cdot \frac{28}{8}\left[4 x+\frac{1}{4}(-y)\right] .
\end{aligned}
$$

This last inequality implies that

$$
|E x-E y|<\frac{1}{2} \cdot \frac{28}{8}|| F x-F y \mid .
$$

Thus condition (A) and hence condition (ii) of Theorem 2.3 is satisfied if we take $\alpha=\frac{28}{8}$. Here $x=0$ is the only point of coincidence of $E$ and $F$.

EXAMPLE (3.3). We illustrate Theorem 2.5 by the following example.

Let $X=R$ and $C=[-1,1]$. Define $E: C \rightarrow C$ by setting

$$
E x= \begin{cases}-x & \text { if } 0 \leqslant x \leqslant 1 \\ -0.9 x & \text { if }-1 \leqslant x<0\end{cases}
$$

Then for $1 \geqslant x \geqslant 0$, we have $E^{2} x=E(-x)=0.9 x$, while for $-1 \leqslant x<0$, one gets $E^{2} x=E(-0.9 x)=0.9 x$. Therefore, for any $x, y \in C$, we get

$$
\left|E^{2} x-E^{2} y\right|=0.9|x-y| \leqslant|x-y| .
$$

Furthermore, for $x, y \in[0,1]$, we have

$$
|E x-E y|=|x-y| \leqslant 1.9|x-y|,
$$

whereas for $x, y \in[-1,0)$

$$
|E x-E y|=0.9|x-y| \leqslant 1.9|x-y| .
$$

Next, we discuss for $x \in[0,1]$, and $y \in[-1,0)$ with the hypothesis $-y \leqslant x$. So we can write

$$
\begin{aligned}
|E x-E y| & =x+0.9(-y) \leqslant x+0.9 x \\
& =1.9 x=\frac{1.9}{2} \cdot(2 x)=(1.9) \frac{1}{2}|x-E x| .
\end{aligned}
$$

If $-y>x$, we agree like foregoing examples. From $-y>x$, it follows that $-y>0.1 x$ which implies $0.9 y-1.9 y>2 x-1.9 x$. From this inequality, we deduce $1.9(x-y)>2 x+0.9(-y) \geqslant x+0.9(-y)$ and therefore

$$
|E x-E y|=x+0.9(-y)<1.9(x-y) \text {. }
$$

Thus condition (ii) of Theorem 2.5 is satisfied if we take $\alpha=1.9$. Now it remains only to verify the inequality $\alpha \delta^{-1}(1-1 / \alpha)<4$, where $\delta$ is the modulus 
of convexity which we may assume as an strictly increasing function $\delta:[0,2] \rightarrow$ $[0,1]$ (see [5]) defined by $\delta(\varepsilon)=\varepsilon / 2$. So $\delta^{-1}:[0,1] \rightarrow[0,2]$ satisfies $\delta^{-1}(\varepsilon)=2 \varepsilon$. Then we obtain

$$
\alpha \delta^{-1}\left(1-\frac{1}{\alpha}\right)=\frac{19}{10} \cdot 2 \cdot\left(1-\frac{10}{19}\right)=\frac{19}{10} \cdot 2 \cdot \frac{9}{19}=\frac{9}{5}<4 .
$$

Therefore the mapping $E$ satisfies all the conditions of Theorem 2.5. Clearly, $E$ has a fixed point namely $x=0$.

\section{Acknowledgement}

We are grateful to Professor S. Sessa for his useful criticism and advice all along.

\section{References}

[1] F. E. Browder, 'Nonexpansive nonlinear operators in a Banach space', Proc. Nat. Acad. Sci. U.S.A. 54 (1965), $1041-1044$.

[2] V. W. Bryant, 'A remark on a fixed point theorem for iterated mappings', Amer. Math. Monthly 75 (1968), 399-400.

[3] Lg. B. Ciric, 'A generalization of Banach's contraction principle', Proc. Amer. Math. Soc. 45 (1974), 267-273.

[4] K. Goebel, 'An elementary proof of the fixed point theorem of Browder and Kirk', Michigan Math. J. 16 (1969), 381-383.

[5] K. Goebel and E. Zlotkiewicz, 'Some fixed point theorems in Banach spaces', Colloq. Math. 23 (1971), 103-106.

[6] G. E. Hardy and T. D. Rogers, 'A generalization of a fixed point theorem of Reich', Canad. Math. Bull. 16 (1973), 201-206.

[7] K. Iséki, 'Fixed point theorem in Banach spaces', Math. Sem. Notes Kobe Univ. 2 (1974), 11-13.

[8] W. A. Kirk, 'A fixed point theorem for mappings which do not increase distances'; Amer. Math. Monthly 72 (1965), 1004-1006.

[9] V. Klee, 'Convex bodies and periodic homeomorphism in Hilbert spaces', Trans. Amer. Math. Soc. 74 (1953), 10-43.

[10] S. Réich, 'Some results concerning contraction mappings', Canad. Math. Bull. 14 (1971), 121-124.

\section{Department of Mathematics}

Aligarh Muslim University

Aligarh-202001

India 